\title{
Activation of a Co-located Meeting by Visualizing Conversation and Facilitating Participant's Behavior*
}

\author{
Hiroyuki ADACHI ${ }^{\dagger \ddagger}$, Seiko MYoJIN ${ }^{\dagger \S}$ and Nobutaka SHIMADA ${ }^{\dagger}$
}

\begin{abstract}
Although there are a lot of opportunities to have a multi-party conversation such as decisionmaking meeting and brainstorming, we do not always have a good communication. In multi-party conversation, it sometimes ends up as one-way conversation because of someone's too much speaking, and people cannot understand a less-speaking person's thought. Considering the situations where a balanced participation is desirable, the amount of speaking time should be well controlled. We propose a co-located meeting support system called ScoringTalk that activates conversation by visualizing conversation state in real-time, providing scores based on the amount of conversation, and facilitating participant's behavior such as speaking, listening, and changing a conversation partner to make a balanced conversation. In ScoringTalk, each participant has a tablet with dual camera, and it works as both sensor and display. Tablets are commonly used in social meetings, therefore, there are no need to prepare projectors and microphones. The evaluation result showed that our system measured conversation, provided the visualization of conversation, scores, and facilitation, and increased the balance of participation while keeping the amount of speaking time.
\end{abstract}

\section{Introduction}

Although there are a lot of opportunities to have a conversation such as meeting and brainstorming, we cannot always have a good communication. In an ideal communication, everyone reaches an acceptable conclusion, and understands each other. On the other hand, in a real communication, it sometimes ends up as one-way conversation because of someone's too much speaking, and people cannot understand a less-speaking person's thought. Considering the situations where a balanced participation is desirable, such as brainstorming and decision-making meeting, the amount of speaking time should be well controlled [4,5,11,15].

We have developed a co-located meeting support system called ScoringTalk that gamifies conversation based on the amount of speaking time and listening time for balancing of participation[1]. It measures a conversation through the tablets with front and back cameras, and scores each participant's speaking individually. The score increases and decreases by the rules of the game; the score increases when a participant speaks and listens, and decreases when a participant speaks too much. In the system, participants

\footnotetext{
* Manuscript Received Date: October 19, 2016

$\dagger$ College of Information Science and Engineering, Ritsumeikan University; Kusatsu, Shiga 525-8577, JAPAN

* Presently with Yahoo Japan Corporation.

$\S$ Presently with Osaka University.

Key Words: CSCW, meeting support, visualization, facilitation, gamification, tablet.
}

have a conversation while trying to maximize their scores, and it makes a balanced conversation.

In this paper, we propose scoring methods for enhancing group activity for individual utterance and mutual conversations. We also propose a facilitation method to increase the variety of opinions for having a better conversation by making all the participants to have a chance to speak. The facilitation suggests next speaking and listening behavior to each participant based on a current state and history of the conversation, for example, encouraging a participant to give an opinion, suppressing too much speaking, and changing a conversation partner. It is because that some participants did not know what behavior would be made a conversation better only with the visualization of conversation and the scores in our earlier experiments. We expect this system activates a conversation and improves the quality of the meeting.

The following are the main contributions of this study.

- Proposes a meeting support system called ScoringTalk that activates conversation by visualizing conversation in real-time, providing scores based on the amount of conversation, and facilitating participant's speaking/listening behavior to make a balanced conversation.

- Develops a tablet system that measures utterance of each participant by using tablet's front and back cameras simultaneously. It measures the position and orientation of the participant's face and estimates the speaking state by im- 
age processing, because the tablet's microphone contains noise of the fan and other participants' voice. The system integrates observations from each participant's tablet, and provides real-time visual feedback on each tablet.

- Evaluates the performance of ScoringTalk in a small group meeting with and without the feedback (visualization and facilitation), and shows that such feedback affects the social dynamics of the conversation.

\section{Related Work}

The visual feedback systems with a shared table using microphones and projectors are popular. Bergstrom et al. [2] proposed Conversation Clock that visualizes personal cumulative speaking time and the duration of the current turn on the table. Ohshima et al.[3] proposed a tabletop system called TableTalkPlus that visualizes the dynamics of communication by crowd of lights. It motivates the participant to talk, changes the direction of the conversation, and designs the field of conversation. Terken et al.[4] proposed a tabletop system that represents amount of speaking time and listening time as circles projected in front of the participants. The size of the circles increases when a person is speaking and listening. It gives a positive feedback about speaking and listening behavior.

The visual feedback systems with wall displays are also popular. DiMicco et al.[5] proposed a system that shows bar chart indicating relative amount of speaking time on a shared display so that the participants can know they are over participated or under participated. Tausch et al.[6] presented Groupgarden which is a metaphorical group mirror providing feedback about individual as well as group performance. The system changes its visualization based on the participation, for example, the petals of the flower fill up when a participant contributes ideas. Takabayashi et al.[7] proposed a discussion support system using visualization of utterance intentions for group decision making.

There are other interaction systems using handheld devices. Meeting Mediator[8] is a portable system that detects social interactions using electronic sensing device and provides visual feedback on a mobile screen for enhancing group collaboration. Fujita et al.[9] proposed Ambient Suite which is a roomshaped information environment that enhances communication in a standing party situation. In this system, people have a cup-shaped device and ware some sensors, and the sensors measure participant's speaking time, hand acceleration, and head rotation. They represented the atmosphere's activity using a combination of these cues.

The visual feedback systems for co-located multiparty conversation are popular (e.g. $[2,3,5])$, however, our system provides not only visualization but also scores for enhancing conversation with gamification and facilitation messages for suggesting participants' speaking/listening behavior. Terken et al.[4] proposed a visual feedback based on participants' speaking time and listening time. Their system has only positive feedback that the size of projected circles increases along with the increase of the amount of speaking/ listening time.

In the literature of discussion analysation and supporting, it is thought to be desirable that all of the participants give opinions for increasing the variety of opinions, not only from a particular participant in decision-making meeting and brainstorming, so that the participation of the group members should be well controlled. Bachour[15] pointed out and verified that member's unbalanced paticipation leads decreasing of motivation in collaborative learning. DiMicco[5], Terken[4] and Schiavo[11] all together mentioned that balancing paticipant's total utterlance time is important for collaborative decision making, which is an opinion-convergent type discussion, not only for brainstorming which is an opinion-divergent one. Recently facilitator, who coordinates an active discussion, becomes popular and has an important role in discussion management. Bachour[15] pointed out that facilitators should have a skill to "ensure that all team members contribute" based on Burns[16].

For balancing each member's paticipation, it is necessary not only to promote someone's speaking, but also to change a dominant participant's behavior. Therefore, our system employs positive and also negative feedback. It scores balance of participation based on rules of the game: the score increases on a balanced conversation, otherwise the score decreases. Groupgarden[6] has a negative feedback, however, the feedback of the system is controlled by a moderator by Wizard of $\mathrm{Oz}[10]$, and actually the system does not provide any automatic feedback by itself. Schiavo et al.[11] proposed an automatic facilitation system using Kinect. It monitors the group members' nonverbal behavior and promotes balanced participation by giving targeted directives to the participants based on a current state of conversation.

On the other hand, our system measures the participants' verbal behavior: how long who speaks to whom and when, and records the history of such information. Our system automatically provides the facilitation suggesting participants' speaking/listening behavior based on not only a current state of conversation but also a history of conversation. The positive and negative feedback messages can lead conversations not only to be more balanced but also conversely to focus a specified participant for sharing his/her opinions more.

In addition, our system has the advantage of being easy to use, because the system only requires tablets or smartphones, and they work as both sensor and display. Tablets/smartphones are one of the handheld devices used in the previous researches[8,9], however, which are more popular than those devices and com- 


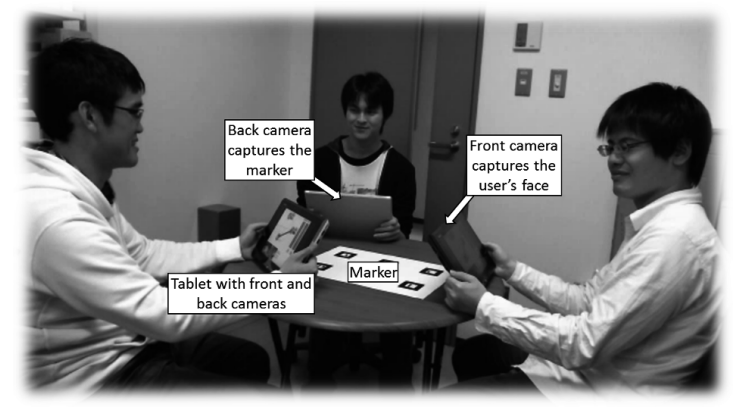

Fig. 1 A group conversation using ScoringTalk. A conversation is monitored through the tablets while the system provides the real-time feedback on the tablets' screens

monly used in social meetings for displaying meeting materials. There are no need to prepare other special things such as projectors, microphones, and sensors. Therefore, the system can be set up easier than most of the previous visual feedback systems.

\section{ScoringTalk}

\subsection{Concepts}

We propose a meeting support system called ScoringTalk that activates co-located small group meeting by providing feedback about balance of conversation. Fig. 1 shows a three-person conversation using ScoringTalk. People have tablets with front and back cameras and talks around a table which has a marker on it. A conversation is monitored through the tablets with dual camera, and the system provides real-time feedback on the tablets' screens.

The concepts of the system are the following.

- Sensing and visualizing device for multiparty conversation

We propose a system using tablets which are popular in today and commonly used in social meetings. Tablets often have dual camera (a front camera and a back camera), and the system uses both cameras simultaneously for sensing the conversation and provides visual feedback on each tablet's screen. There is no need to prepare special things such as microphones and projectors while most of previous works requires such things for measurement and visualization, therefore our system has the advantage of being easy to use. In addition, the system can be used in a conversation where participants may need to move since the participant and the device are together.

\section{- Incentive design for multi-party conver- sation}

We introduce a game element into the conversation to motivate a participant and control a balance of amount of conversation. We propose a scoring method based on the amount of user's speaking time and listening time for balancing

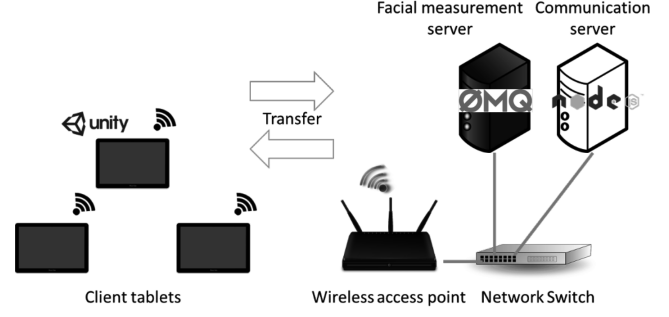

Fig. 2 System structure. The system consists of tablets, a communication server, and a facial measurement server. They transfer messages via wireless

of participation. We expect that providing a real-time feedback of the score encourages the participants to speak more and gives the participants chance to consider lack of balance of participation. We also propose a facilitation method for helping a behavior of a participant based on a current participation and a transition of conversation. It enables a participant to consider a concrete behavior for a better conversation and makes a conversation more active.

\subsection{Implementation}

We implement the system as shown in Fig. 2. We use Windows 8.1 tablets and develop the application for them with Unity ${ }^{1}$. A communication server is running on Node.js ${ }^{2}$ and a facial measurement server is running over ZeroMQ ${ }^{3}$.

In the following section, we describe the methods for measuring the conversation, for scoring based on the amount of conversation, for visualizing conversation, and for facilitating participants' behaviors.

\subsubsection{Measurements}

In this section, we describe the methods for measurement of individual utterance and for measurement of group conversation as a whole.

There are three pieces of information of individual utterance: who speaks, when speaks, and from where to where. Each tablet obtains them in the following way by using its front and back cameras, and sends them to the communication server in every frame.

(1) Who speaks: Each user has a tablet and is connected to the communication server via wireless with an ID. A current speaker is specified by this ID.

(2) When speaks: A user's mouth movement is captured by the front camera of the tablet which the user has. When the change rate of user's mouth open level exceeds the predetermined threshold, the system recognizes that the user is speaking. If the user's face is not found, the system understands the user is not speaking. The balanced error rate of the speak detection in the ideal condition is about $10 \%$.

\footnotetext{
${ }^{1} \mathrm{~A}$ platform for creating games and interactive experience.

${ }^{2}$ A server-side JavaScript environment.

${ }^{3} \mathrm{~A}$ cross-platform, cross-language messaging library.
} 


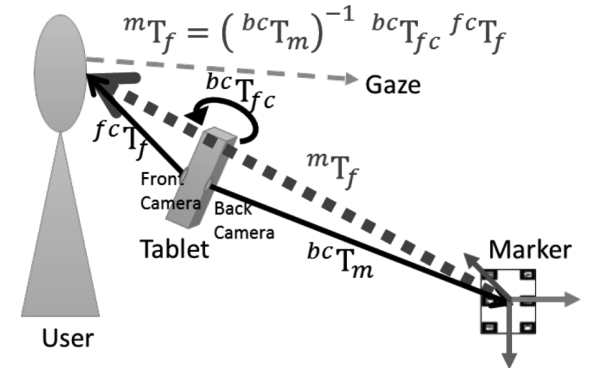

Fig. 3 Geometric relation of user-tablet-marker

(3) From where to where: A user's position and the face direction in the world coordinate determined by markers on the table are calculated from the captured images by the front and back cameras of the tablet and geometric relation of user-tablet-marker (see Fig. 3). The homogeneous transformation matrix ${ }^{m} \mathrm{~T}_{f}$ represents the above information. It is obtained by multiplying the following three matrices as

$$
{ }^{m} \mathrm{~T}_{f}=\left({ }^{b c} \mathrm{~T}_{m}\right)^{-1 b c} \mathrm{~T}_{f c}{ }^{f c} \mathrm{~T}_{f} .
$$

First, ${ }^{b c} \mathrm{~T}_{m}$ is measured from a back camera image by using NyARToolkit ${ }^{1}$. Second, ${ }^{b c} \mathrm{~T}_{f c}$ is calibrated in advance since the relative position of the two cameras on a particular tablet is fixed. Last, ${ }^{f c} \mathrm{~T}_{f}$ is measured from a front camera image by using OKAO ${ }^{\circledR}$ Vision[12]. The accuracy of the estimation of the face direction in horizontal has a margin of error of plus or minus two degrees[13]. Tomioka et al. proposed a pseudo see-through tablet by employing the front and back cameras in the similar framework[14].

The system specifies who exists where, where he/she faces to, and when he/she speaks as described above. The system integrates these observations on the server, and estimates a conversation partner of each user through the following steps (see Fig. 4).

(1) Calculate a vector $U_{i}$ as a face direction of user $i$.

(2) Calculate a vector $V_{i j}$ which directs from user $i$ to user $j$

(3) Calculate a similarity of $U_{i}$ and $V_{i j}, \operatorname{Sim}_{i j}$, as;

$$
\begin{aligned}
\theta & =\cos ^{-1} \frac{U_{i} \cdot V_{i j}}{\left\|U_{i}\right\|\left\|V_{i j}\right\|}, \\
\operatorname{Sim}_{i j} & = \begin{cases}\cos \theta & \text { if }|\theta|<\theta_{\text {thresh }} \\
0, & \text { otherwise, }\end{cases}
\end{aligned}
$$

where $\theta_{\text {thresh }}$ represents a maximal angular difference between $U_{i}$ and $V_{i j}$ when user $i$ stares to user $j$ for speaking or listening to him/her. Here $\theta_{\text {thresh }}$ is experimentally fixed as $\pi / 6$ by considering measurement error and is independent of the number of participants.

(4) Select a user $j$ with maximum $\operatorname{Sim}_{i j}$ as a conversation partner of the user $i$. If $\operatorname{Sim}_{i j}=0$ then

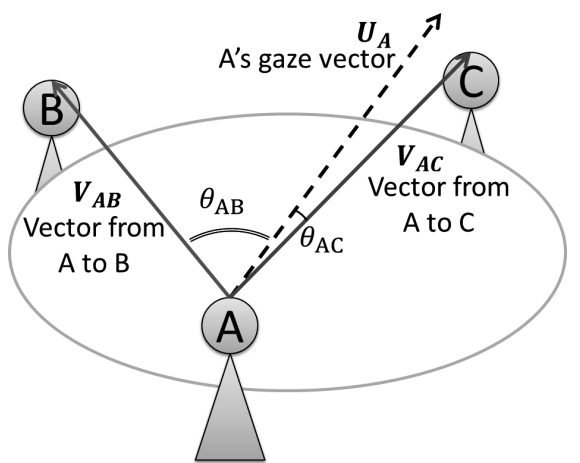

Fig. 4 Conversation partner estimation. The conversational partner is decided by the participants' position and the face direction

the user $i$ has no conversation partner.

Finally, the system obtains how long a person speaks to the other person and how long the person watches him/her as the statistical profiles of conversation.

\subsubsection{Scoring}

In this section, we present two scores in brainstorming for activating the group activity. We designed two indices for evaluating group activity with the following considerations;

- Maximize the amount of individual utterance. We consider active participation leads idea generation.

- Balance the amount of pair conversation. We consider having a conversation with variety of people increases the variety of ideas.

We define the index of balance of individual utterance as Eq.(3), applying Gini coefficient as a measure of equality. Similar metrics inspired by the Gini coefficient have already been adopted for measuring participation equality in group conversations $[4,11]$.

$$
I_{\text {indiv }}^{(t)}=\frac{n}{2(n-1)} \sum_{i}^{n} \mid \text { Utterance }_{i}^{(t)}-\frac{1}{n} \mid .
$$

Utterance $_{i}^{(t)}$ is a relative amount of utterance of user $i$ at time $t$, and $n$ is the number of users. $I_{\text {indiv }}^{(t)}$ becomes 0 where the amounts of individual utterances are balanced, and becomes 1 where they are unbalanced. We also define the index of balance of pair conversation as Eq.(4), as similar as Eq.(3).

$$
I_{\text {pair }}^{(t)}=\frac{{ }_{n} \mathrm{C}_{2}}{2\left({ }_{n} \mathrm{C}_{2}-1\right)} \sum_{i, j} \mid \text { Conversation }_{i j}^{(t)}-\frac{1}{{ }_{n} \mathrm{C}_{2}} \mid \text {.( }
$$

Conversation $_{i j}^{(t)}$ is a relative amount of pair conversation between user $i$ and user $j$ at time $t$, and ${ }_{n} \mathrm{C}_{2}$ means the number of combination of two participants.

The indices are updated on every frame based on the measurements obtained within 30 seconds. We calculate the weighted average of each index in time window $T$, and convert it to the score with a scale of $0 \%$ to $100 \%$ as Eqs.(5),(6).

\footnotetext{
${ }^{1} \mathrm{~A}$ library for AR application.
} 


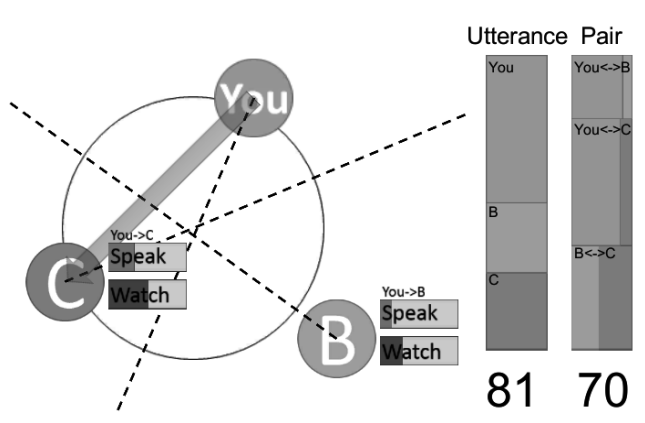

Fig. 5 The visual feedback on users' devices with annotations. The left area shows the visualization of the conversation The right area shows the scores and bar charts representing group activity

$$
\begin{gathered}
\text { Score }_{\text {indiv }}^{(t)}=100\left(1-\frac{\sum_{s=1}^{T} s I_{\text {indiv }}^{(t-T+s)}}{\sum_{s=1}^{T} s}\right) . \\
\text { Score }_{\text {pair }}^{(t)}=100\left(1-\frac{\sum_{s=1}^{T} s I_{\text {pair }}^{(t-T+s)}}{\sum_{s=1}^{T} s}\right) .
\end{gathered}
$$

The system provides these scores to the users in real-time through the tablets' screens so that the users can control their speaking.

\subsubsection{Visualization}

Fig. 5 shows an example of the visual feedback. The left area shows a visualization of conversation. It represents a situation where user $A$ is speaking to user $C$. A circle represents a user's position, and a dotted line represents a user's face direction. A purple bar beside a circle represent a cumulative speaking time from a user to another, and a red bar represents a cumulative watching time. The right area shows the group activity. The bar charts represent the relative amount of individual utterances and pair conversations, and the below numbers show those scores.

This visualization makes the users understand their amount of conversation, and may provide motivation for them, for example, to speak to someone who has not so much talked with. The visualization is currently on table-centric view, we also investigate a usercentric view visualization.

\subsubsection{Facilitation}

In this section, we present the facilitation mechanism of ScoringTalk in three-person conversation. This facilitation aims to control the amount of conversation for increasing participation of all. The facilitation messages are provided by the following steps. First, the system labels each participant every frame with the three states of a balance of participation:

Over-participated A participant's relative speaking time is more than $50 \%$.

Under-participated A participant's relative speaking time is less than $20 \%$.

Almost equal Otherwise (a participant's relative speaking time is between 21-49\%).

Second, the system determines a state of the group based on the result of labeling of the participants. If there is an over-participated person, then the state of the group is over-participated, else if there is an underparticipated person, then the state of the group is under-participated, otherwise, the state of the group is almost equal. Last, the system provides the following facilitation messages every 30 seconds depending on the state of the group as shown in Fig. 6 .

Over-participated In this case, the system tries to make a less-speaking participant have a chance to speak and control an over-participated person's speaking. The system provides a message, "How about to be a listener?" to the participant who labeled over-participated. The system provides a message, "Let's share your idea!" to the other participants.

Under-participated In this case, the system tries to encourage a less-speaking participant to speak and propose the other participants to have a conversation with him/her. We consider there are two ways to have a conversation: making an opportunity to converse with by speaking to him/her or hearing from him/her. The system provides a message, "Let's share your idea!" to the participant who labeled under-participated. The system randomly choices and provides the one of the messages, "How about speaking to (a under-participated person's name)?" and "How about hearing the idea of (a under-participated person's name)?" to the other participants.

Almost equal In this case, the system tells the participants that the conversation has been well balanced and suggests the participants to change conversation partners for increasing the variety of opinions. The system randomly choices and provides the one of the messages, "OK! Keep it up!" and "How about speaking to (a name of a person who has not talked with so much)?" to all the participants.

Schiavo et al.[11] proposed a facilitation system based on a balance of participation like this, however their facilitation is based on a current state of conversation. Our system obtains a current state of conversation: how long who speaks to whom in a recent time span, and history of conversation: how long who speaks to whom and when and the amount of conversation time. Therefore, the system can not only promote of speaking but also suggest a participant for having a conversation with the other participant who has not talked with so much.

By providing the facilitation messages, the participants will be able to understand the timing of speaking and listening for a better conversation, while the participants may not understand what the behavior will be made a better conversation where the system providing only the visualization of conversation. In addition, the facilitation gives a participant chance to notice that the participant has been talking only a specific participant, and the participant will be able to change his/her behavior to speak to the other par- 

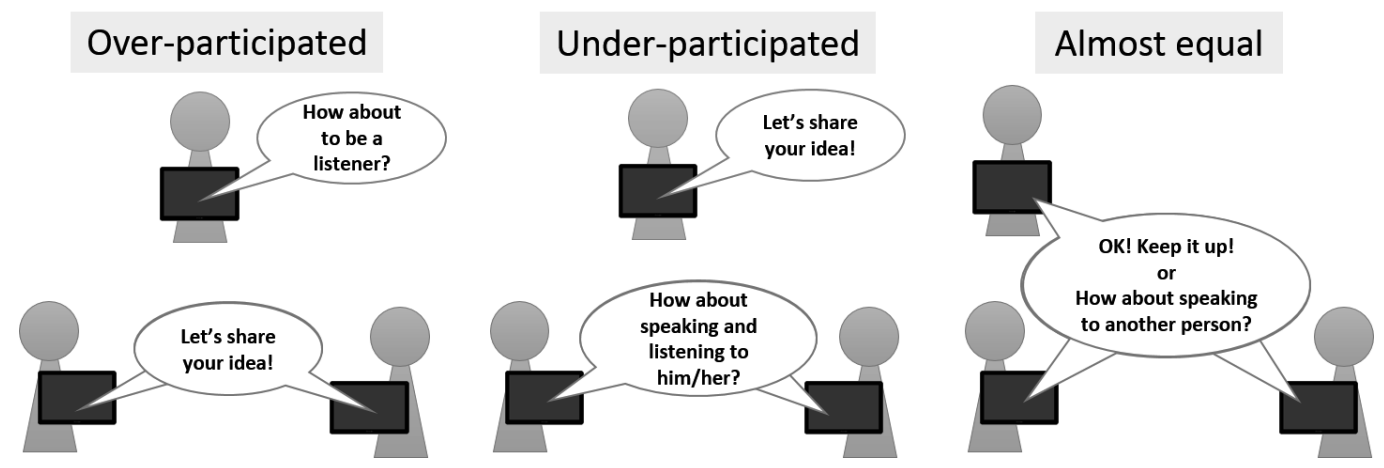

Fig. 6 Facilitation messages on the three states of a balance of participation

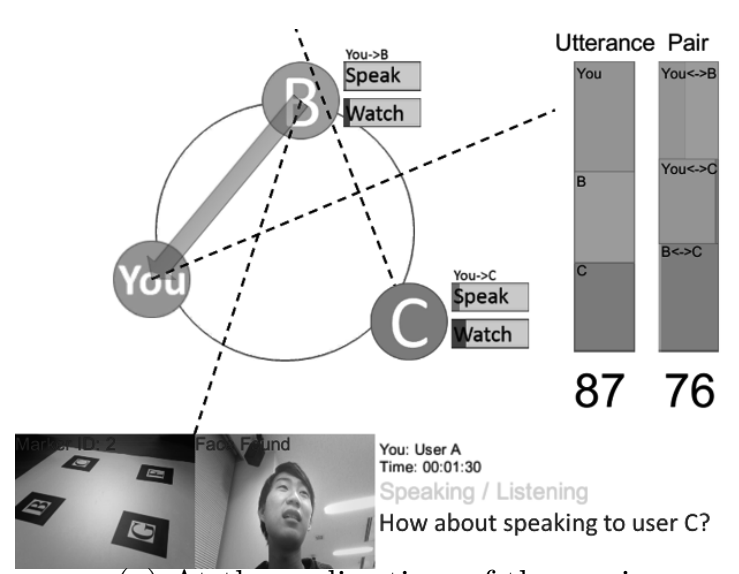

(a) At the earlier time of the session

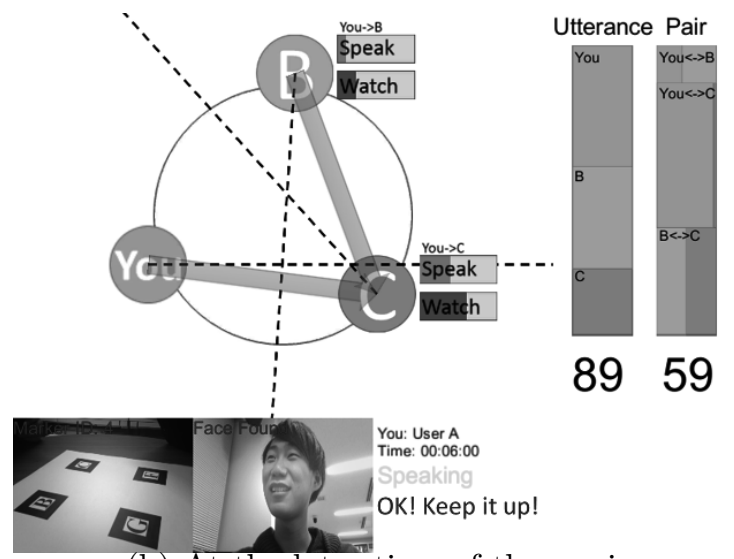

(b) At the later time of the session

Fig. 7 The examples of the screenshot of tablet interface ticipant.

Fig. 7(a) shows an example of the screenshot of tablet interface at the earlier time of the session and Fig. 7(b) shows an example of the screenshot of tablet interface at the later time of the session. The facilitation messages are showed in the lower-right of the interface as blue texts. The two messages are different, and they show that the system provides the facilitation messages based on the situation at the time.

\section{Evaluation}

We evaluated ScoringTalk to examine whether it can balance participation and what was the effect of the feedback. In this section, we describe the set-up, the results, and the discussion of the test.

\subsection{Methods \\ 4.1.1 Participants}

To evaluate the system, we conducted a study of four groups of three participants each, a total of 12 participants (11 males, 1 female, 22-25 years old). All the participants were the graduate or undergraduate students of the author's laboratory and all of them already knew each other.

\subsubsection{Experimental Task}

To verify the effectiveness of the system, we performed a within-subjects experiment. Each group participated in two discussion sessions. In each discussion, the members had to express opinions on a particular topic. In one discussion session, participants obtained feedback (with feedback condition); in the other condition, the feedback were not provided (without feedback condition).

With feedback condition The system provides feedback about the scores and the facilitation messages on the participants' tablets.

Without feedback condition The scores and facilitation messages are not provided.

Fig. 8 shows the visualization on both conditions. The scores on the upper-right and the facilitation messages on the lower-right are disappeared from the visualization on without feedback condition.

\subsubsection{Procedure}

At the start of the experiment, participants had an instruction of usage of the system; how to measure a speaking state and the meaning of the visualization. Then the group first had a warm-up practice for about three minutes in order to get used to the system. After the warm-up practice, the participants had seven minutes conversation for each of two topics: Topic 1) "Where is the best location do you think for a new convenience store?" and Topic 2) "What goods do you want to handle at a convenience store?", with and without feedback. Topic 1 is about the selection of the best choice from discussion, which can be classified as opinion-convergence type. Topic 2 is about the pick up of several possiblities, which can be classified as opinion-divergence type. The opinion-convergence 


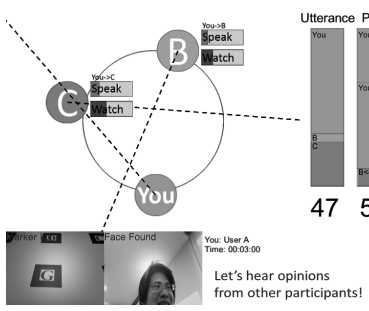
condition (a) With-feedback

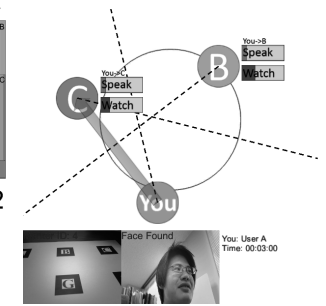

(b) Without-feedback condition
Fig. 8 The difference of the visualization of both conditions

type discussion tends to be lead by an opinion leader and thus other participants may hesitate or feel difficulty to present their opinions. Therefore we targeted Topic 1 session to balance the user utterances by giving feedback messages to the participants. Topic 2 session is regared as the baseline to evaluate the effect of the feedback facilitation. To avoid order effects, the order of conditions was balanced across groups.

After two conversation role-plays, the participants were asked to fill in a questionnaire. In the experiment, we used three tablets (Sony VAIO Duo 11 x2, Microsoft Surface Pro $3 \times 1$ ) and a PC with Intel Core i7 $3.4 \mathrm{GHz}$ as a server.

\subsubsection{Evaluation Metrics}

We evaluate the effectiveness of the system in the following three metrics.

(1) Average balancing degree of amount of utterance: The system provides the balacing scores (Eqs.(5),(6)) to the particiants for balancing the amount of utterance based on gamification. It is desirable that the average score on with-feedback condition is higher than that on without-feedback condition since it suggests that the participants keep their participations balanced. Therefore, we compare the transition of the scores between with-feedback condition and without-feedback condition.

(2) Average total speaking time: Providing feedback of balancing degree may disturbs natural conversations of participants because the balancing feedback will too much suppress the participants speaking. We compare the difference of total speaking time between with-feedback and without-feedback condition to measure this negative effect.

(3) Results of four types of questionnaires: First, we compare the average scores of the questionnaire on 7-scale in the two conditions: withfeedback and without-feedback (the result will be shown in Fig. 12). Second, we analyze participant's behavior when he/she is given a facilitation message to change a conversation target (the result will be shown in Fig. 13). Third, we analyze what element was fun for the participants on with-feedback condition (the result will be shown in Fig. 14). Last, we evaluate what

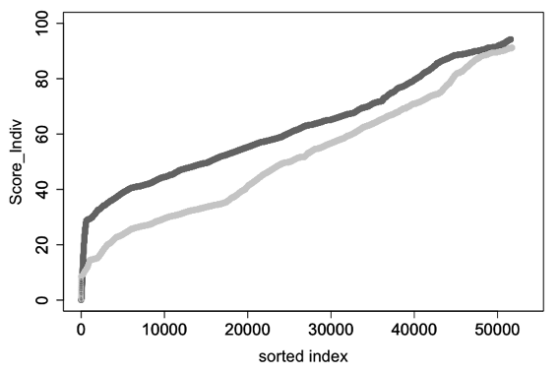

Fig. 9 Individual utterance scores per frame sorted in ascending order for all 8 discussion sessions (red: with-feedback, green: without-feedback)

items displayed on the tablet are often looked at and whether the items are useful or not (the result will be shown in Fig. 15).

\subsection{Results and Discussion}

\subsubsection{The Scores and Amount of Conver-} sation

Four groups participated in the evaluation experiment. Figs. 16-23 show the score transitions for 8 experimental discussion sessions (2 discussion topics by 4 groups). For each figure, the upper graph shows the utterance ratio of each user over the session time. The lower graph shows the two scores of individual utterance (red line) and pair conversation green line). The score value has 0 to 100 and the higher value means that the utterance and conversation were higher balanced.

For more clear comparison between with- and withoutfeedback, we show Fig. 9 in which all scores of individual utterance per time frame are sorted in ascending order. The red line is for with-feedback and green one is for without-feedback session. It is apperently shown that the scores of individual utterance withfeedback are higher than ones without-feedback. We also verified the effect by statistical way, Wilcoxon's rank-sum test and then it was shown that the difference is significant with $\mathrm{p}$-value $<10^{-6}$.

On the other hand, the improvement of the scores of pair conversation was not proven. The scores per frame sorted in ascending order like Fig.9 is shown as Fig.10, in which the scores for with-feedback (red line) and ones for without-feedback intersects each other and Wilcoxon's test's p-value is approximately 0.5 .

Fig. 11 shows the difference of total speech time amount between with- and without-feedback conditions. The left-hand and center graphs represent the average total speaking time of each participant for both conditions. The right-hand graph represents the average difference of total speech times for each user. The figure shows the total speech time is a nearly equal amount for both conditions. We could not confirm that the total speaking time increases on with feedback condition, however at least, it suggests that the feedback cacilitation message did not 


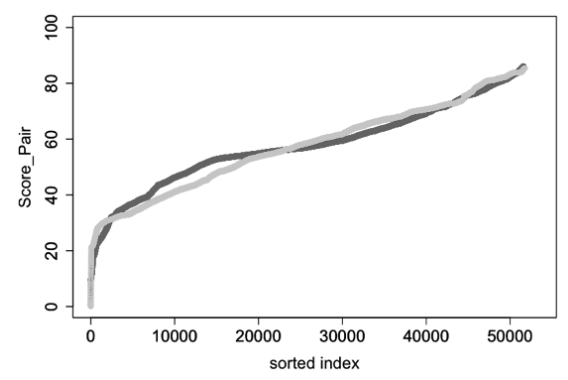

Fig. 10 Pair conversation scores per frame sorted in ascending order for all 8 discussion sessions (red: with feedback, green: without feedback)

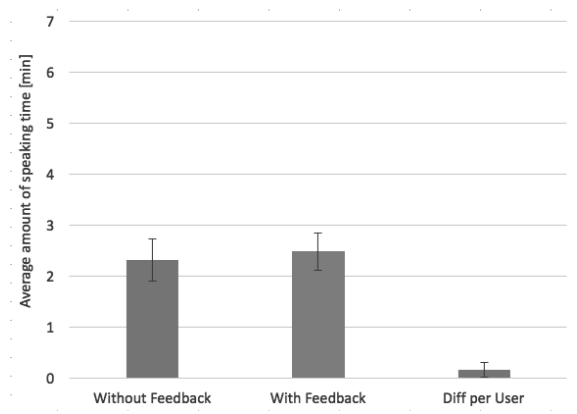

Fig. 11 Amount of speaking time of with and without feedback: left) average total time per user, right) average difference of them. Bars mean SD

supresse user's speaking oppotunity. The balance degree of utterance amount on with-feedback condition was higher than that of without feedback condition, and the values of the average amount of speaking time on both conditions have about the same amount, therefore the feedback could balance the utterance amount without giving negative effects to the total speaking time.

\subsubsection{Questionnaire and Interview}

Fig. 12 shows the result of the questionnaire in 7-point Likert scale with and without feedback conditions. A Wilcoxon signed-rank test indicates that Question 1 (I found that controlling amount of utterance was easy), Question 5 (I felt that I could concentrate on the conversation), and Question 6 (I felt to change a conversation partner) are statistically significant at the $5 \%$ level. The results of Question 1 and Question 6 show that the feedback works well. According to the users' comments on the questionnaire, there are some positive impressions for them, for example, "I could have the balance of participation on my mind on with feedback condition." On the other hand, the result of Question 5 shows that the feedback makes negative impression for the participants, for example, one of the participants felt that "I could not speak in timing of my choice because my attention moved to the scores." It suggests that the feedback may put a restriction on the participant's behavior. We will need to work to improve this point.

On Question 6, 11 of 12 participants answered that they felt to change a conversation partner with

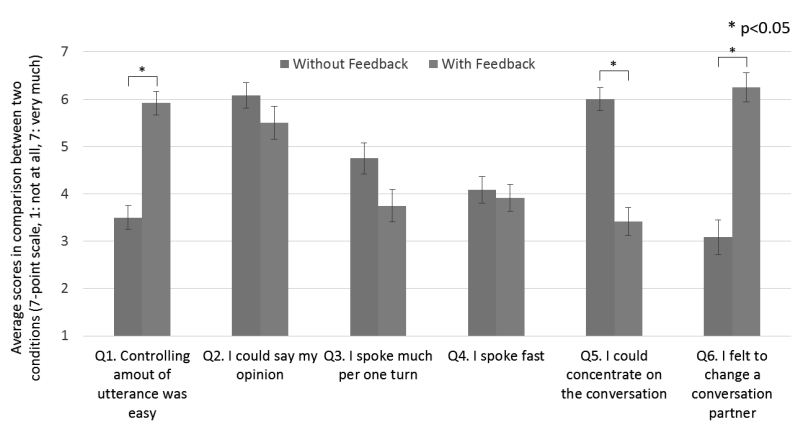

Fig. 12 Average scores in comparison between withfeedback and without-feedback condition

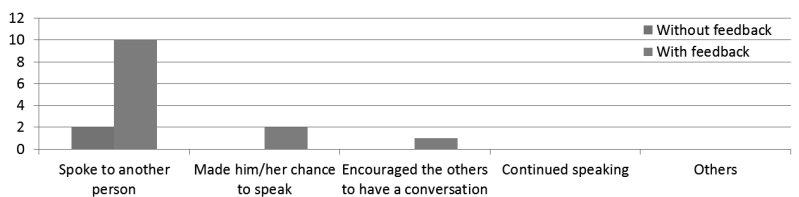

Fig. 13 The answer of the question about the participant's behavior when he/she was given a facilitation message to change a conversation target

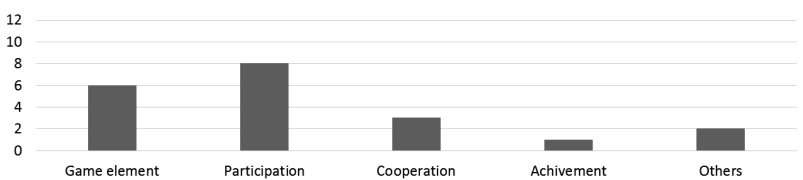

Fig. 14 The answer of the question about fun of the conversation on with feedback condition

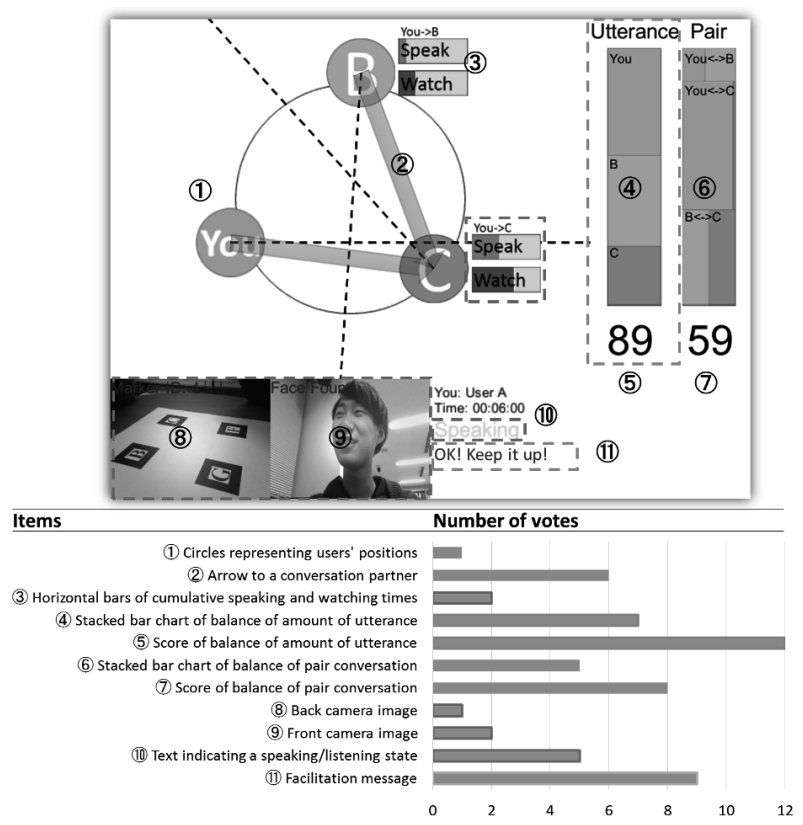

Fig. 15 Number of votes for interface items often looked at (green frame: useful, red frame: not useful)

feedback, and 1 of 12 participant answered that he did not feel so. Fig. 13 shows the questionnaire result about the participants' behavior when they tried to change a conversation partner. The most of the participants spoke to another person when they felt to change a conversation partner on with feedback con- 


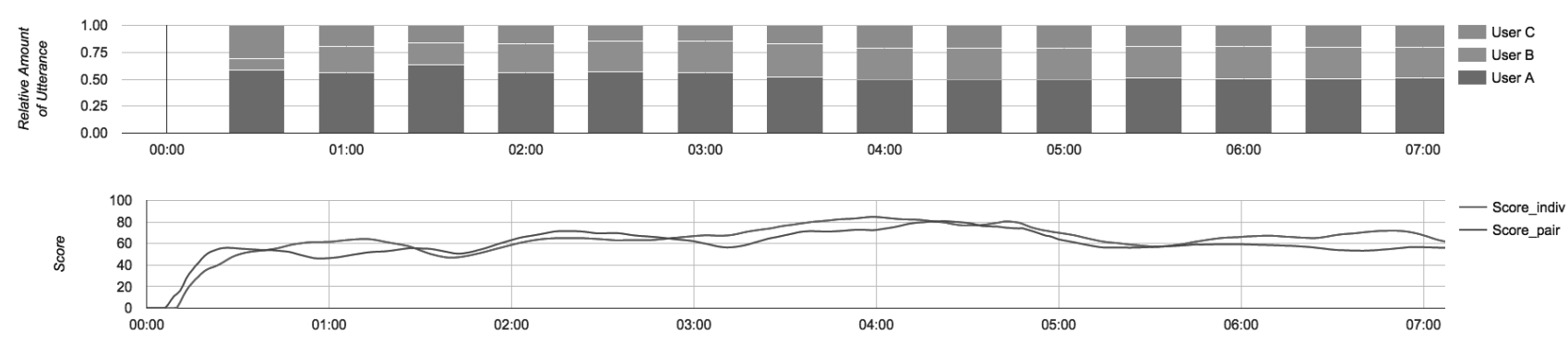

Fig. 16 Score transition for Group 1 (Topic 1 with FB)
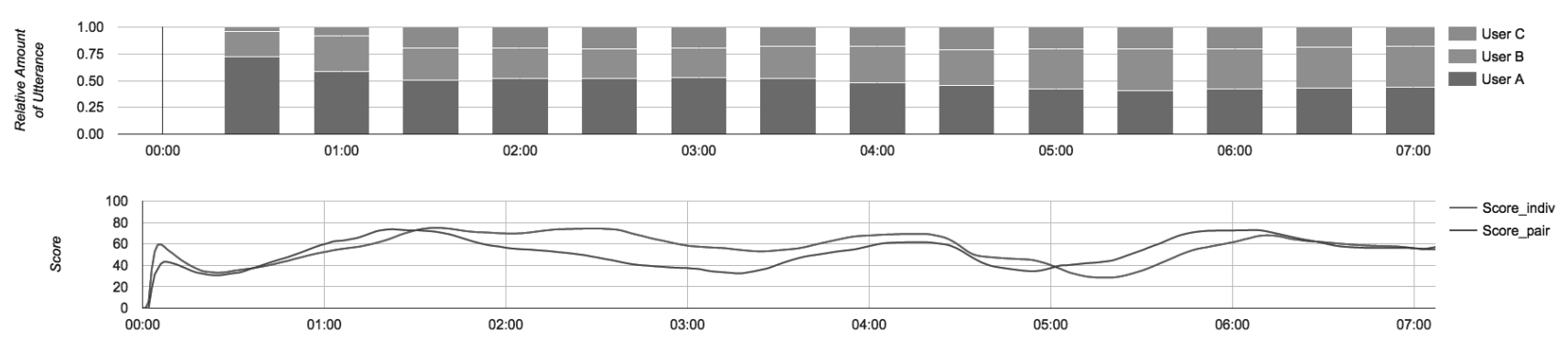

Fig. 17 Score transition for Group 1 (Topic 2 without FB)
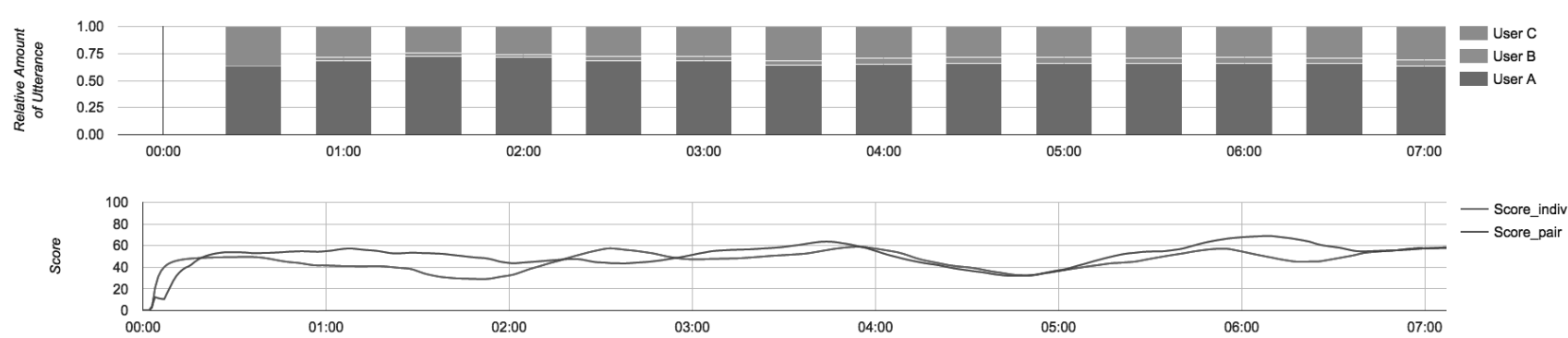

Fig. 18 Score transition for Group 2 (Topic 1 with FB)
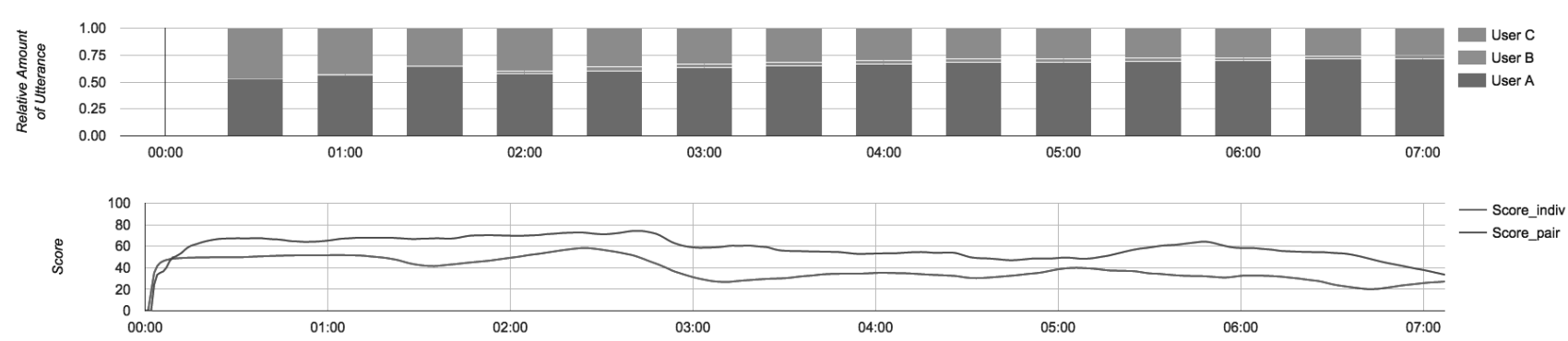

Fig. 19 Score transition for Group 2 (Topic 2 without FB)
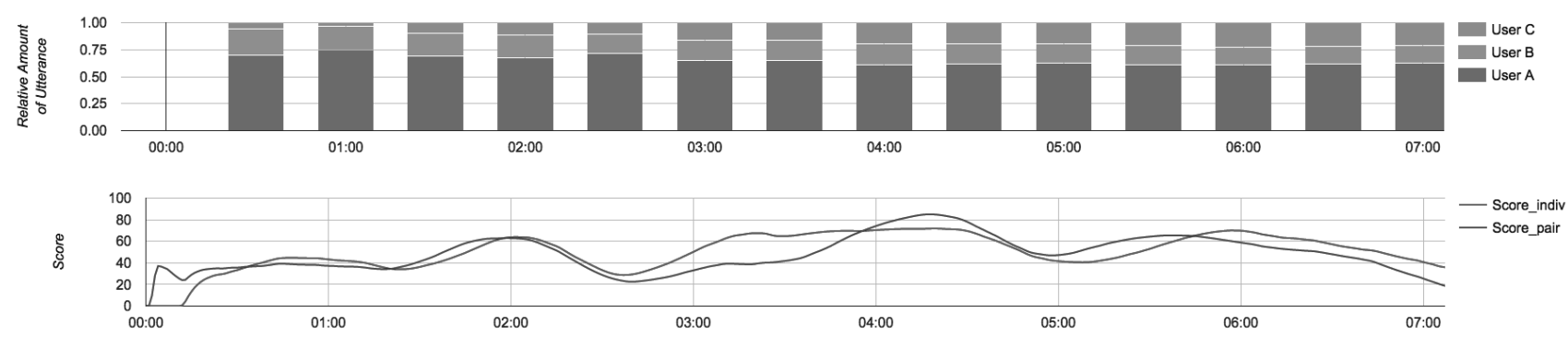

Fig. 20 Score transition for Group 3 (Topic 1 with FB) 


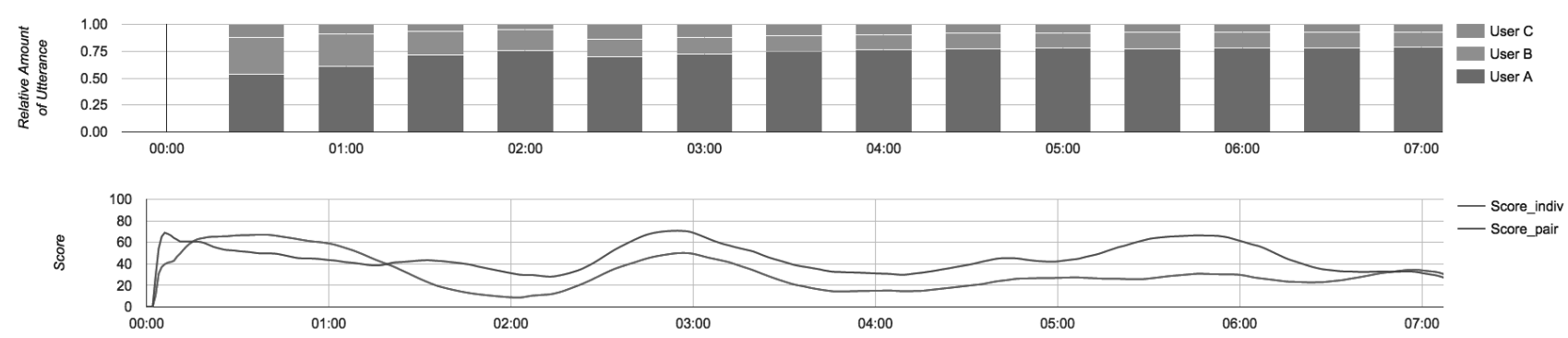

Fig. 21 Score transition for Group 3 (Topic 2 without FB)
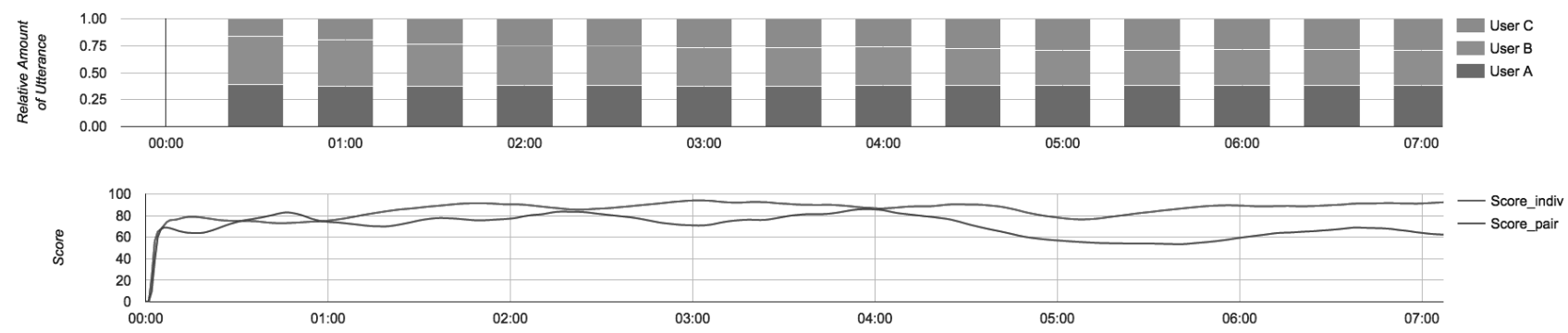

Fig. 22 Score transition for Group 4 (Topic 1 with FB)

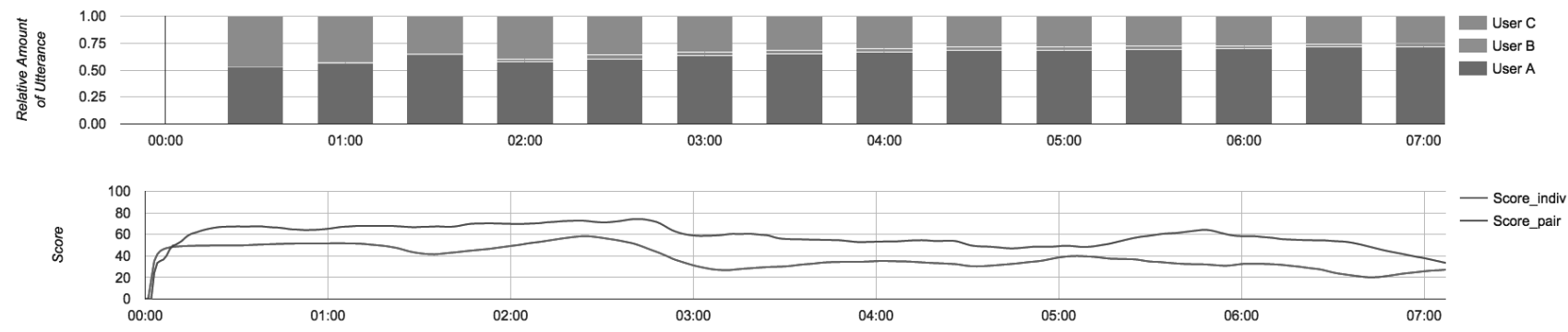

Fig. 23 Score transition for Group 4 (Topic 2 without FB)

dition. Some of the participants made the other participants chance to speak and encouraged the other participants to have a conversation. The result suggests that the feedback have enough effect to provide facilitation for changing the participants' behavior.

Fig. 14 shows the questionnaire result about fun of conversation on with feedback condition. It shows that 8 of 12 participants felt that it was interesting that their behavior made the scores change, and it also shows that 6 of 12 participants felt that it was interesting that the conversation had game elements, for example, increasing/decreasing of the scores and bar charts. Two participants choose "Others", and answered that "The advice from the system was useful," and "It was helpful that I could take my conversation objectively." However, there are some negative impressions, for example, "I could not concentrate on the conversation when I tried to aim at the high score." We hope to make improvements to make the participants more interesting in scoring since the current system just shows the scores.

Fig. 15 shows the number of votes for interface items which the participants were often looking at. The green frames represent that these items were useful, and the red frames represent that these items were not useful. Most of the participant were interested in "Score of balance of amount of utterance" and "facilitation message", and answered that these feedback were useful. There are some positive impressions, for example, "I felt that I could hear the others' opinions since the system provides facilitation message", on the other hand, some participant felt that they could not concentrate the conversation when they tried to aim at the high score. The participants answered that "horizontal bars of cumulative speaking and watching times", "front camera image", "back camera image", and "text indicating a speaking/listening state" were not useful.

The results of the questionnaire suggest that the system with scoring for group and facilitation makes participants easier to control the amount of utterance and also makes participants have a chance to change a conversation partner. However, there are some negative impressions caused by the feedback, it need to be examined the method for feedback of the scores and the facilitation messages. There can be possible improvements for those problems like iconizing frequently shown messages or suppression of repeating once displayed messages for a while. 


\section{Conclusion}

We proposed ScoringTalk that supports activation of co-located meeting based on a balance of amount of conversation. Most of previous computer-supported meeting systems are difficult to set up because they require microphones and projectors. On the other hand, our system is easy to use since each participant has a tablet and it measures and visualizes a conversation. The system not only measures and visualizes a conversation, but also provides the scores with game element and provides facilitation for participant's behavior. We evaluated the system with and without feedback. The results showed that the proposed method has an effect for balancing of participation based on the balance degree of amount of conversation. The questionnaire results also showed that the participants felt the effect of the feedback for facilitating of behavior and for controlling of timing of utterance. However, there are some negative impressions that the participants could not concentrate on the conversation because of paying attention to the feedback, we need to improve it.

In this paper, we researched only the three-person conversation, the effectiveness of the feedback for fouror-more-person conversation should be researched. The current facilitation method depends on a three-person conversation, we should expand it for such a conversation. In addition, all the participants in the experiment were the students. The unbalanced participation is likely to occur in a conversation with people in different position such as teachers and students. We also would like to use the system in such a situation, and evaluate the effectiveness of the system. Besides, we consider our system can be applied to education and training systems for learning a way of discussion. Participants can know their amount of conversation through the visualization and can control the timing of speaking and listening by the scores and facilitation messages. By continuously using the system in discussion, it may improve the ability of conversation time management and facilitation skills of the participants. We would like to evaluate the system from this viewpoint in the future.

\section{Acknowledgments}

This work was supported by JSPS KAKENHI Grant Number $15 \mathrm{H} 02764$ and by the foundation for the Fusion of Science and Technology. The authors would like to thank the OMRON Corporation let us use the $\mathrm{OKAO}^{\circledR}$ Vision Library in their courtesy.

\section{References}

[1] H. Adachi, S. Myojin and N. Shimada: ScoringTalk: A tablet system scoring and visualizing conversation for balancing of participation; SIGGRAPH ASIA 2015 Mobile Graphics and Interactive Applications, ACM, pp. 9:1-5 (2015)

[2] T. Bergstrom and K. Karahalios: Conversation clock:
Visualizing audio patterns in co-located groups; System Sciences, 200\%. HICSS 200\%. 40th Annual Hawaii International Conference on, IEEE, pp. 7878 (2007)

[3] N. Ohshima, K. Okazawa, H. Honda and M. Okada: TableTalkPlus: An artifact for promoting mutuality and social bonding among dia logue participants; Journal of Human Interface Society, Vol. 11, No. 1, pp. 105-114 (2009) (in Japanese)

[4] J. Terken and J. Sturm: Multimodal support for social dynamics in co-located meetings; Personal and Ubiquitous Computing, Vol. 14, No. 8, pp. 703-714 (2010).

[5] J. M. DiMicco, A. Pandolfo and W. Bender: Influencing group participation with a shared display; Proceedings of the 2004 ACM Conference on Computer Supported Cooperative Work, ACM, pp. 614$623(2004)$

[6] S. Tausch, D. Hausen, I. Kosan, A. Raltchev and H. Hussmann: Groupgarden: supporting brainstorming through a metaphorical group mirror on table or wall; Proceedings of the 8th Nordic Conference on Human-Computer Interaction: Fun, Fast, Foundational, ACM, pp. 541-550 (2014)

[7] T. Takabayashi, Y. Kamiya, K. Kunieda and K. Yamada: A discussion support system using visualization of utterance intentions for group decision making; IEICE Technical Report, Human Information Processing, Vol. 111, No. 60, pp. 43-48 (2011) (in Japanese)

[8] T. Kim, A. Chang, L. Holland and A. S. Pentland: Meeting mediator: enhancing group collaboration using sociometric feedback; Proceedings of the 2008 ACM Conference on Computer Supported Cooperative Work, ACM, pp. 457-466 (2008)

[9] K. Fujita, Y. Itoh, H. Ohsaki, N. Ono, K. Kagawa, K. Takashima, S. Tsugawa, K. Nakajima, Y. Hayashi and F. Kishino: AmbientSuite: enhancing communication among multiple participants; Proceedings of the 8th International Conference on Advances in Computer Entertainment Technology, ACM, p. 25 (2011)

[10] J. F. Kelley: An empirical methodology for writing user-friendly natural language computer applications; Proceedings of the SIGCHI Conference on $\mathrm{Hu}$ man Factors in Computing Systems, ACM, pp. 193196 (1983)

[11] G. Schiavo, A. Cappelletti, E. Mencarini, O. Stock and M. Zancanaro: Overt or subtle? Supporting group conversations with automatically targeted directives; Proceedings of the 19th international conference on Intelligent User Interfaces, ACM, pp. 225234 (2014)

[12] OMRON Corporation: Image Sensing Technology OKAO Vrsion; http://www.omron.com/ecb/ products/mobile/ (accessed 2016-01-17)

[13] H. Adachi, S. Myojin and N. Shimada: Tablet system for sensing and visualizing statistical profiles of multiparty conversation; Consumer Electronics (GCCE), 2014 IEEE 3rd Global Conference on, IEEE, pp. 407411 (2014)

[14] M. Tomioka, S. Ikeda and K. Sato: Approximated user-perspective rendering in tablet-based augmented reality; Mixed and Augmented Reality 
(ISMAR), 2013 IEEE International Symposium on, IEEE, pp. 21-28 (2013)

[15] K. Bachour, F. Kaplan and P. Dillenbourg: An interactive table for supporting participation balance in face-to-face collaborative learning; IEEE Transaction on Learning Technologies, Vol. 3, Issue 3, pp. 203-213 (2010)

[16] G. Burns: The secrets of team facilitation; Training E Development, Vol. 49, No. 6, pp. 46-53 (1995)

\section{Authors}

\section{Hiroyuki ADACHI}

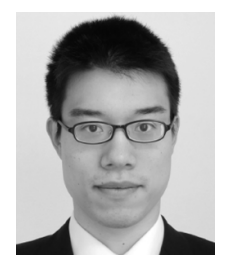

He was born in 1991. He received the B.E., M.E. degrees from Ritsumeikan University in 2014 and 2016. He is now an employee of Yahoo Japan Corporation.

\section{Seiko Myojin (Member)}

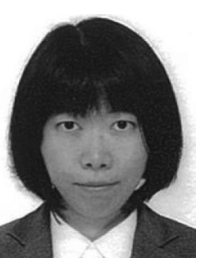

She is a specially appointed researcher at Osaka University. She is a member of IPSJ, VRSJ, HIS, ISCIE, and The Society for Art and Science. She was previously a tokunin assistant professor at Ritsumeikan University. Before joining Ritsumeikan University, she was a specially appointed researcher at Osaka University and experienced a short internship at NTT Cyber Space Laboratories. She obtained a doctorate from Osaka University. She was also a special research student at Nara Institute of Science and Technology over a period of time

\section{Nobutaka Shimada (Member)}

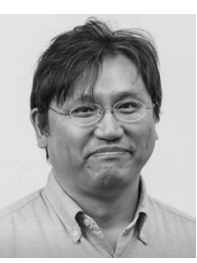

He received the B.E., M.E. and Ph.D (Engineering) degrees from Osaka University in 1992, 1994, and 1997 respectively. He was an assistant of Graduate School of Engineering, Osaka University from 1997 to 2002. In 2003, he was an associate professor of Graduate School of Engineering, Osaka University. In 2004, he moved to College of Information Science and Engineering, Ritsumeikan University and is currently a professor (2011-). In 2007, he was engaged in research as a visiting associate professor of the Robotics Institute, Carnegie Mellon University. His research interest includes computer vision, gesture interface and interactive robots. He is a member of IEEE, IEICE, IPSJ, JSAI, HIS, ISCIE, and RSJ. 